\title{
La marque odonto-légale de la momie de Grenoble
}

\section{RÉSUMÉ}

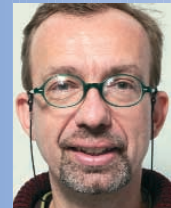

Francis JANOT

Professeur à la faculté d'odontologie de Nancy 1 ,

Ancien membre scientifique

de I'IFAO du Caire,

Expert près la cour d'appel de Nancy,

Faculté d'odontologie de Nancy 1 ,

96, avenue de Lattre de Tassigny,

BP 50208

54004 Nancy cedex.

\section{Jean-Paul ARTIS}

Professeur à la faculté d'odontologie de Nancy 1 ,

Président de la Compagnie

des experts judiciaires

près la cour d'appel de Nancy.

\section{Henry COUDANE}

Doyen de la faculté de médecine de Nancy 1 ,

Responsable de l'Unité d'enseignement de médecine légale et de droit médical.

Pierre BRAVETTI

Maître de conférence,

Praticien hospitalier

à la faculté d'odontologie de Nancy,

Doyen de la faculté d'odontologie,

Responsable du département

de chirurgie et pathologie buccale,

Service d'odontologie,

CHU de Nancy.

Par ses spécificités, l'odontologie légale est une discipline incontournable dans l'étude des corps des civilisations anciennes. Dans l'Égypte ancienne, certains indices fournis par l'organe dentaire sont de véritables preuves médico-légales révélatrices d'une profession. Tel est le cas pour la momie de Grenoble.

odontologie légale

- égyptologie

- marque odonto-légale

momie de Grenoble
AOS 2009:248:313-323

DOI: $10.1051 /$ aos/2009406

(C) AEOS / EDP Sciences 


\section{Introduction}

$\rightarrow$ Une double formation - odontologique et égyptologique - permet bien évidemment d'être un interlocuteur privilégié entre les spécialistes médicaux et les conservateurs de musée. C'est ainsi que j'ai pu initier et coordonner, en 2003-2004, au Museo Egizio de Turin, deux études pluridisciplinaires de momies égyptiennes conservées dans les collections dont une momie datée de l'Ancien Empire (IVe dynastie, 2200 avant J.-C.) [1] et les momies de l'architecte Kha et de son épouse Merit, datées du Nouvel Empire (XVIII dynastie, 1500 avant J.-C.) [2, 3].

Fort de cette expérience, un nouvel axe de recherche s'amorce maintenant à la faculté d'odontologie de Nancy avec les momies des collections muséales françaises. Le but est simple : il s'agit de se rapprocher au plus près de la vie quotidienne de ces anciens Égyptiens.
Ainsi, en mars 2008, le corps d'une femme surnommée la «prophétesse», conservée dans les salles du muséum de Grenoble et provenant de la ville d'Antinoé en Moyenne-Égypte, a subi une étude tomodensitométrique dans la Clinique universitaire de radiologie et d'imagerie médicale de I’hôpital A. Michallon.

L'examen macroscopique associé aux études anthropologiques d'identification permet de lui attribuer une quarantaine d'années au moment du décès.

L'analyse des reconstructions multiplanaires et tridimensionnelles, qui est encore en cours au Laboratoire lorrain de recherche en informatique et ses applications (LORIA, D. Fass/équipe Mosel), permettra peut-être de diagnostiquer la cause de sa mort.

\section{Examen bucco-maxillaire}

La momie est retrouvée la bouche largement ouverte. Celle-ci n'est pas obstruée par des linges comme cela se pratique habituellement au moment de sa purification à partir de la Basse Époque [4] (fig. 1).

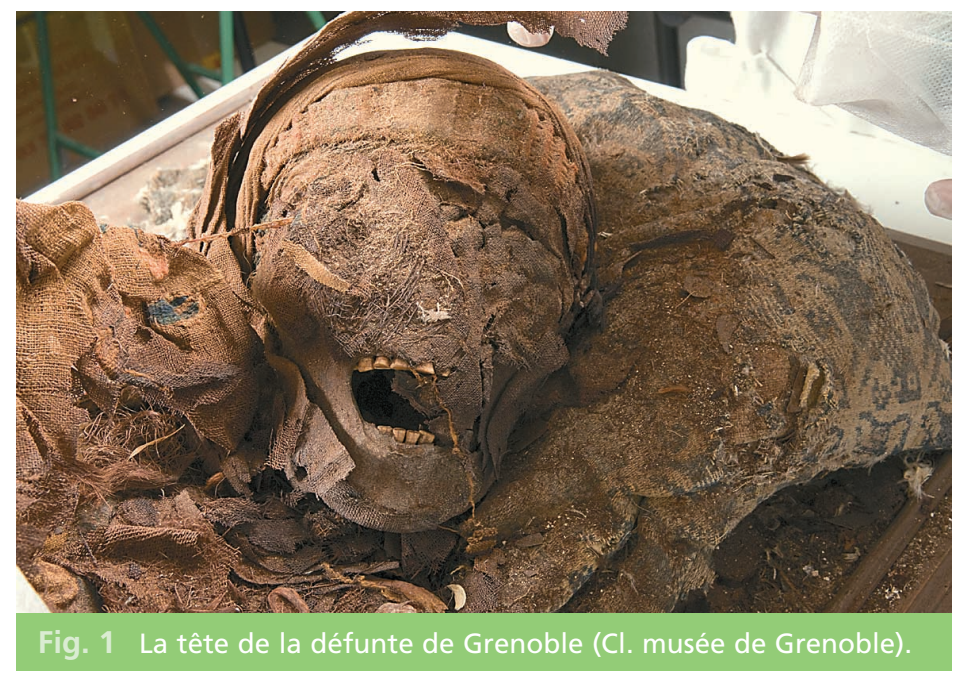




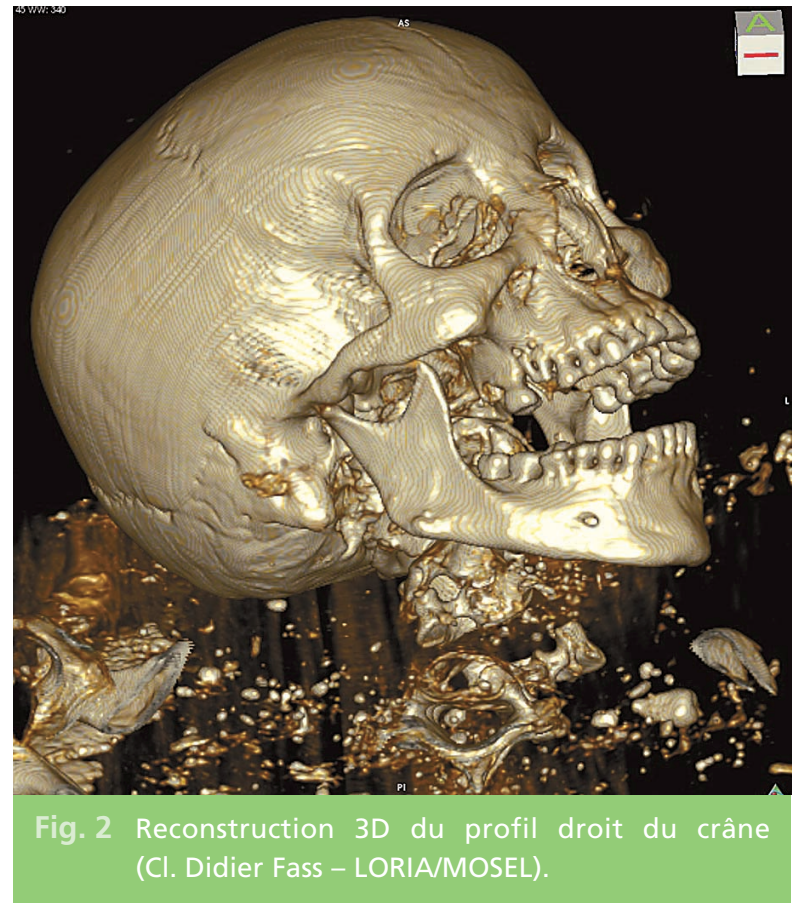

D'ores et déjà, l'analyse maxillo-faciale sur les reconstructions 3D permet de mettre en évidence, à la mandibule, une exostose latérale droite au niveau de l'insertion gogniaque du muscle masséter (fig. 2).

L'examen odontologique, rendu possible par la reconstruction 3D et les filtres appliqués lors du traitement des images, montre, du côté gauche, l'inclusion de la canine inférieure (33) ainsi que la perte ante mortem de la deuxième prémolaire (35) et de la première molaire (36).

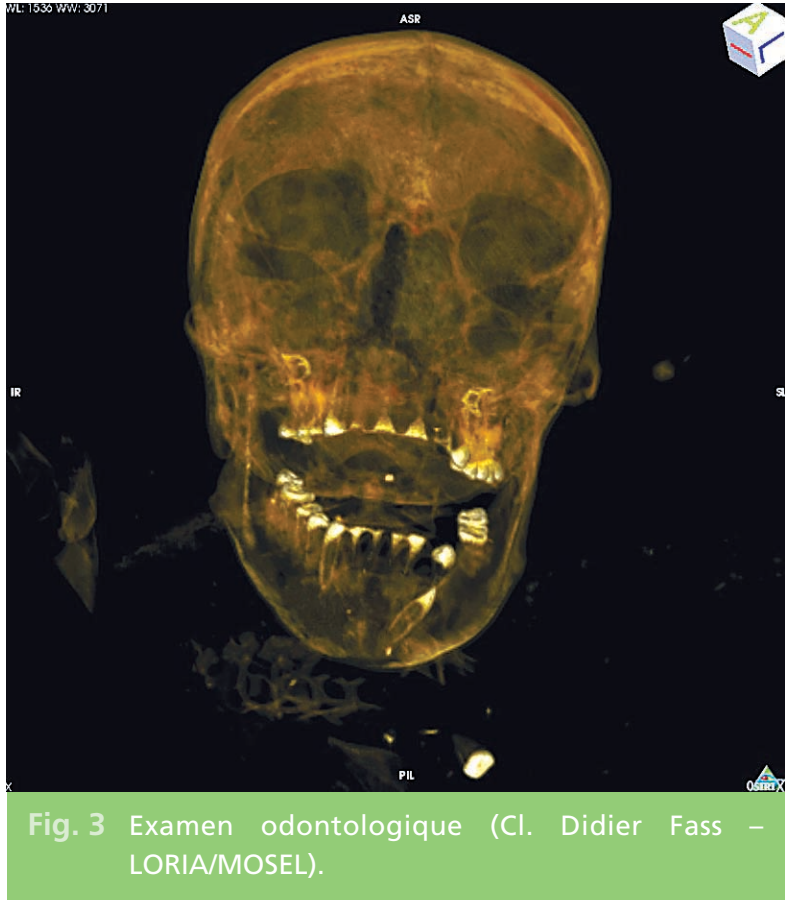

L'os apparaît parfaitement cicatrisé à ce niveau (fig. 3).

Du côté droit, la première molaire supérieure (16) ne subsiste plus qu'à l'état de racines. La présence de kystes radiculaires à leurs apex signe la chronicité de l'infection. L'alvéole de la troisième molaire (18) est vide. Pourtant, la dent est retrouvée intacte, à la gauche de la $5^{\mathrm{e}}$ vertèbre cervicale, sur la planche de fond du sarcophage sur laquelle repose encore le corps.

\section{La marque odonto-légale}

L'examen attentif de la denture fait apparaître une anomalie d'abrasion des bords libres au niveau du groupe incisif $(11,12,21,22)$ parfaitement indépendante de toute mastication [5]. Une rainure, de direction mésio-distale, marque les surfaces coronaires. De plus, il existe une continuité de cette anomalie qui débute à partir de l'incisive latérale (22) pour se terminer au point de contact distal de l'incisive latérale (12).

En vue palatine, l'usure des surfaces de contact est plus ou moins marquée selon la dent observée. La dentine exposée forme un schéma de mastication dû à des frottements multiples sur 


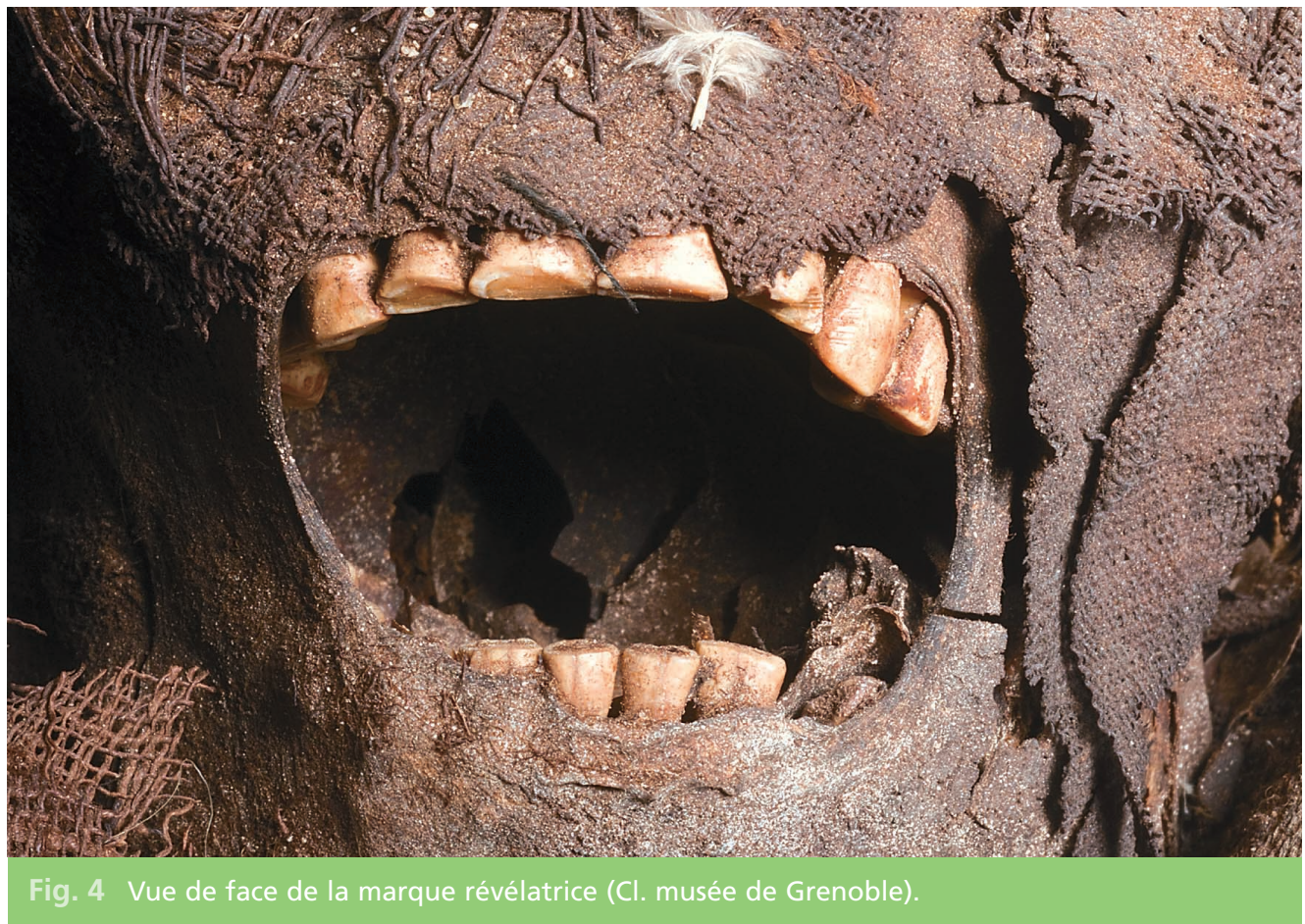

une surface dure introduite transversalement dans la bouche.

La face vestibulaire de la canine gauche (23) porte une zone de stries parallèles disposées les unes au-dessus des autres qui se continuent dans la couronne littéralement creusée de l'incisive latérale (22).
Ces traces permettent de restituer un mouvement d'interposition d'un objet entre les dents qui commence du côté gauche de la denture. II permet également d'affirmer que la défunte était à prédominance gauchère (fig. 4).

\section{Identification de l'objet inséré}

Bien évidemment, il semble absolument indispensable d'identifier le type d'objet introduit transversalement dans la bouche de la défunte, capable de laisser une telle «empreinte» médico-légale. Pour cela, nous devons la comparer avec d'autres marques décrites lors d'activités professionnelles connues et parfaitement individualisées.

La découverte d'un luth dans la tombe de la défunte indique une activité de musicienne. Si celle-ci avait utilisé un instrument à vent de l'Antiquité tardive, du type aulos, I'abrasion n'aurait touché que les incisives centrales maxillaires comme cela se diagnostique de nos jours [6]. La marque n'a donc rien à voir avec une quelconque activité de musicienne.

Elle aurait pu confectionner des vanneries et des cordes. En effet, l'examen de vestiges dentaires, retrouvés lors de fouilles archéologiques, décrit l'existence de profondes rai- 
nures au contour net sur les faces occlusales des dents antérieures [7]. De plus, ces marques professionnelles reconnues creusent en profondeur les seules faces occlusales de l'incisive et de la canine. Dès lors, une activité de travail de vannerie et de cordes peut être écartée.

De même, l'activité de la défunte laisse une marque totalement différente de celle décrite sur le groupe incisif maxillaire de la cordonnière mise au jour sur la pyramide du roi Pépy ler à Saqqarah [8, 9]. Un travail de cordonnier peut également être éliminé.
Enfin, il faut envisager les pathologies de type bruxomanie qui surviennent lors d'une habitude vicieuse nocturne [10]. Dans ce cas, les abrasions des cingulums incisifs supérieurs laissent toujours des facettes d'abrasion sur les incisives inférieures. Ces dernières ne sont pas retrouvées dans le cas de la défunte de Grenoble.

De surcroît, il est également possible d'éliminer une abrasion résultant d'un comportement observable chez une patiente anorexique-boulimique [11].

\section{Interposition d'une racine?}

Aux XIX-XXe siècles, les ethnies Kôngo/Vili et Yombé possèdent de nombreuses statuettes en bois de chefs et de devins. Représentés assis ou debout, ces derniers mâchent éternellement la racine d'une plante (fig. 5).
Il existe également des sceptres en ivoire dont l'extrémité est ornée d'une représentation de chef qui effectue la même action. La racine est tenue fermement de la main gauche ou droite [12].

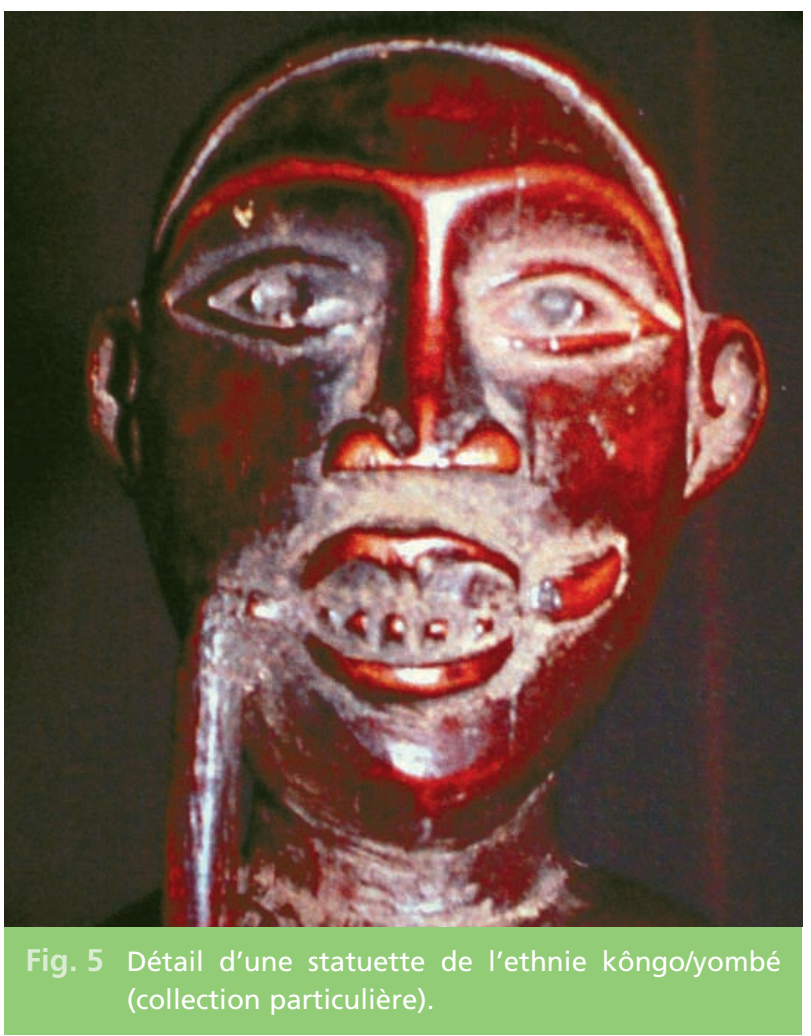


Il apparaît parfaitement crédible de mettre en parallèle la marque révélatrice identifiée sur l'organe dentaire de la défunte du musée de Grenoble avec le mode d'insertion transversal d'une racine.

Les usures et les rainures polymorphes décrites sur les incisives supérieures centrales et latérales ont été faites lors d'un mouvement mandibulaire, effectué d'avant en arrière, qui fait pendant à un mouvement de tirage destiné à réaliser la dilacération des fibres de la racine afin d'en extraire le jus.

L'usure plus importante des surfaces du groupe incisif droit $(11,12)$ est la conséquence de la pression maximale des forces exercées lors de cette dilacération. De surcroît, l'activité du masséter a provoqué des microtraumatismes en traction sur son insertion mandibulaire au niveau de l'angle gogniaque droit. L'exostose observée en serait la conséquence directe.

\section{Les vertus de la racine africaine}

La racine utilisée par les ethnies du Congo est appelée munkwisa. Identifié par les botanistes comme Tabernanthe iboga, cet arbuste contient dans son écorce un alcaloïde, l'ibogaïne, dont les propriétés stimulantes du système nerveux central peuvent entraîner, à forte dose, des hallucinations et des convulsions [13]. Les Africains utilisent ces propriétés hallucinogènes afin d'exacerber les pouvoirs de vision et de clairvoyance. L'initié est alors capable d'entrer en communication avec l'esprit des ancêtres.

\section{Le métier de la défunte}

A. Gayet aurait lu sur un vestige de sa caisse plâtrée son titre : Hémét-nétjer, littéralement «servante divine» [14]. Bien qu'il faille se méfier de la rigueur scientifique du fouilleur, la défunte de Grenoble aurait pu exercer la profession de prêtresse dans la ville d'Antinoé. Outre son activité rituelle quotidienne, son sacerdoce aurait pu comporter un aspect oraculaire complémentaire de "prophétesse», «celle qui parle à la place du dieu».

\section{Le clergé oraculaire dans l'Égypte ancienne}

Les membres du clergé participaient aux procédés de consultation oraculaire lors des processions de la barque du dieu Amon à partir du Nouvel Empire [15]. À Deir el-Médineh, à la $\mathrm{XI} \mathrm{X}^{\mathrm{e}}-\mathrm{Xx} \mathrm{X}^{\mathrm{e}}$ dynastie, les réponses d'Aménophis jer divinisé aux questions des plaignants sont soit orales, soit écrites [16]. Enfin, la pratique «des billets couplés», utilisée dès le ıer millénaire avant J.-C., connaît, après une vague croissante jusqu'au III siècle après J.-C., une longévité attestée, par des papyrus rédigés en copte, jusqu'au vII ${ }^{\mathrm{e}}$-VIII ${ }^{\mathrm{e}}$ siècle après J.-C. [17]. 
De plus, la nécropole Nord d'Antinoé, de laquelle vient la défunte de Grenoble, a livré tout un ensemble de ces «billets couplés» écrits en copte [20]. Les demandes sont précisément adressées au "dieu de Saint Kollouthos», médecin-martyr de la ville. Ainsi, à l'époque copte, il existe un lieu qui conserve le corps du saint. Son monastère devenu centre de culte possède bien un oratoire.

\section{Les techniques oraculaires utilisées}

La documentation papyrologique n'explicite pas les techniques utilisées par les prêtres pour répondre aux questions des plaignants. On sait, par des découvertes archéologiques, que les billets déposés dans un trou creusé directement dans le mur du naos du temple y demeurent la nuit. Le lendemain, la version choisie par le dieu est remise au fidèle.

Les égyptologues accordent à la technique du tirage au sort la règle à laquelle obéit le prêtre au moment du choix du billet. Caché de tous, l'officiant est alors décrit comme «habité par le dieu» [21].

Ne faut-il voir qu'un oracle manipulé par des enjeux de pouvoir et de cupidité ou bien l'influence de croyances impalpables qui autorisent une communication avec les forces du monde surnaturel que notre monde moderne n'est plus à même de comprendre ?

\section{Prêtresse à l'époque copte}

Le prélèvement d'os, effectué dans la diaphyse fémorale gauche, permet, grâce à l'analyse au carbone 14, de dater la défunte de Grenoble au VI-VIIe siècle après J.-C., donc à l'époque copte (Lyon, 4817) et non plus au ॥-IIe siècle après J.-C. Cette attribution à l'époque romaine est réalisée à partir de la datation du matériel archéologique mis au jour par A. Gayet au début du $x x^{e}$ siècle. Il se compose de quatre vases, d'une figure de Déméter en terre cuite, de deux flacons de verre et du seul luth connu dans l'Égypte ancienne. Ils sont actuellement l'objet d'études séparées.

Lors de la description de la momie-cordonnière, la marque révélatrice odonto-légale s'appuyait également sur une preuve philologique associée à une preuve archéologique incontestable.
Dans le cas de la momie de Grenoble, il n'existe ni texte, ni matériel archéologique précis pour étayer ce dossier. Toutefois, l'existence de pratiques oraculaires par la technique des «billets couplés» est démontrée à Antinoé pour l'époque copte. Compte tenu des indices médico-légaux associés aux gestes africains, il semble possible d'inférer que la défunte de Grenoble exerçait la profession de prêtresse avec le passage quotidien entre sa denture d'une racine aux propriétés psychoactives dédiées à la clairvoyance. D'autant que les analyses toxicologiques de ses cheveux sont encore en cours. Elles apporteront peut-être, comme en criminalistique, la preuve indicative ou disculpante de la consommation ou pas d'un produit stimulant [22]. 


\section{Apport fondamental de l’odontologie légale dans cette étude}

La convergence de faisceaux d'indices anatomiques, radiologiques, identifiés, répertoriés puis associés au dossier égyptologique, aboutit à une proposition concernant l'activité professionnelle du corps conservé dans les collections de Grenoble. Celle-ci ne manquera pas d'être âprement discutée par les égyptologues.

D'évidence, la méthodologie qui consiste à analyser des indices puis à démontrer leur relation entre eux et, enfin, à en faire la synthèse, correspond parfaitement à une démarche scientifique moderne pluridisciplinaire qu'il faut maintenant appliquer aux corps anciens ou modernes, découverts dans les musées, les chantiers de fouilles et les scènes de crime.
C'est pourquoi, dès la rentrée universitaire de septembre 2009, la faculté d'odontologie de Nancy 1 supervise la spécialité «criminalistique et archéologie» dans le cadre du master Nancy-Université intitulé : «Éthique de la santé et médecine légale» sous la direction du $\operatorname{Pr} \mathrm{H}$. Coudane, doyen de la faculté de médecine et responsable de l'unité de médecine légale et de droit médical.

Le projet a été rendu possible grâce à une étroite collaboration avec le Dr Pierre Bravetti, doyen de la faculté d'odontologie de Nancy 1 et le professeur Jean-Paul Artis, président de la Compagnie des experts judiciaires près la cour d'appel de Nancy.

REMERCIEMENTS

Nous exprimons toute notre reconnaissance à Armand Fayard, directeur du muséum d'histoire naturelle, Guy Tosatto, directeur du musée de Grenoble, Hélène Vincent, conservateur en chef au musée de Grenoble et Fabienne Cottin, assistante de conservation au musée de Grenoble, pour la confiance qu'elles ont témoignée en autorisant le déplacement et l'étude de la "prophétesse» au CHU de Grenoble sans oublier l'équipe technique et de restauration du musée de Grenoble.

Nos plus vifs remerciements vont au professeur Gilbert Ferretti, chef du service de la clinique universitaire du $\mathrm{CHU}$ de Grenoble, et à son équipe pour leur précieuse et indispensable collaboration à cette première étude pluridisciplinaire.

Nos remerciements s'adressent également aux docteurs Gilles Bourret, JeanFrançois Thiébaut et Hervé Vitte, du Département de prothèse de la faculté d'odontologie de Nancy 1, pour leurs suggestions et conseils si avisés.

Enfin, il nous faut saluer vivement I'anthropologue de terrain Alberto Salza, membre du comité scientifique du Centro Studi Africani del Piemonte, pour ses nombreuses explications sur le continent africain. 


\section{Première nationale à Nancy}

\section{Master «Éthique de la santé et médecine légale»}

Le parcours général du master définit deux spécialités intitulées: «criminalistique et archéologie» et «éthique, communication, expertises et droits de la personne», en regroupant, dans une approche lorraine, la complémentarité des compétences et des savoirs des différentes facultés de Nancy 1 et 2.

\section{$\checkmark$ Objectifs de la formation}

La spécialité «criminalistique et archélogie» propose un enseignement pluridisciplinaire théorique et pratique qui concerne le corps humain retrouvé dans une scène de crime ou d'archéologie.

La spécialité «éthique, communication, expertises et droits de la personne» propose un enseignement pluridisciplinaire théorique et pratique qui concerne les enjeux éthiques des progrès techniques médicaux, les droits de la personne, tout en apportant les outils et les réponses aux questions expertales inhérentes.

- Monde médical : formation des futurs chercheurs et des professionnels de ces disciplines en les rendant aptes à la maîtrise des sciences médico-légales, dans les cadres médico-psychologiques, judiciaires ou archéologiques.

- Monde judiciaire : aptitude à intégrer dans leurs décisions et leurs plaidoiries les différentes disciplines d'expertises scientifiques.

- Monde des lettres : spécialisation pour les acteurs de l'archéologie et de l'anthropologie avec une possibilité de passerelles avec les acteurs du monde judiciaire.

\section{$\checkmark$ Publics concernés}

Cette formation novatrice s'adresse aux étudiants et aux professionnels en activité issus du monde médical (médecins, odontologistes, pharmaciens), judiciaire (magistrats, avocats, officier de police judiciaire et de gendarmerie, TIC, experts judiciaires et de compagnies d'assurances) et des lettres (psychologues, archéologues, anthropologues).

La spécialité «criminalistique et archéologie» (Responsable : Fr. Janot)

Le domaine thématique «criminalistique et archéologie» est en phase directe avec les problèmes de notre époque. Par le fait, la mise en évidence d'un corps et/ou d'un squelette lors d'une fouille archéologique doit se dérouler à l'égal d'une scène de crime contemporaine. Ainsi, les méthodes actuellement employées par les techniciens de l'identification criminelle (TIC) pourraient s'enrichir de cet enseignement dans la recherche d'une scène de crime extérieure (localisée ou non) et d'incendie. De fait, il va s'agir, dès le lieu sécurisé, de retrouver l'ultime position imposée au cadavre de la victime par le(s) criminel(s) ou les circonstances.

D'évidence, les sciences légales et l'archéologie funéraire devenue " légale " sont des disciplines étroitement intriquées.

\section{$\checkmark$ Comment s'inscrire}

Sélection des candidats sur dossier.

\section{$\checkmark$ Pour toute information}

Pascale Rateau

Faculté de médecine - Service de médecine légale et droit de la santé

9, avenue de la Forêt de Haye - BP 184 - 54505 Vandœuvre-lès-Nancy Cedex

Tél : 03.83.68.37.13

Email : Pascale.Rateau@medecine.uhp-nancy.fr

Pour le M1 : les dossiers de candidature sont à télécharger sur le site de la Faculté de médecine :

http://www.medecine.uhp-nancy.fr

Pour le M2 : Les dossiers de candidature sont à télécharger sur le Site de l'université Henri Poincaré, Nancy 1 http://www.uhp-nancy.fr 


\section{Bibliographie}

1. Marochetti EF, Curti $A$, Demichelis $\mathrm{S}$, Janot $\mathrm{Fr}$, Cesarani F, Grilletto R. Le paquet, sépulture anonyme de I'Ancien Empire provenant de Gébélein et conservée au musée égyptien de Turin.

Bulletin de l'Institut français d'archéologie orientale 2003;103:235-256.

2. Marochetti EF, Olivia C, Doneux K, Curti A, Janot Fr. The Mummies of Kha and Merit:

Embalming Ritual and Restauration Work. In: Rabino-Massa E, ed., Proceedings $V$ World Congress on Mummies Studies, J Biol Res Turin: 2005;//80:243-247.

3. Janot Fr.

Momies.

Rituels d'immortalité dans l'Égypte ancienne. Paris :

White Star 2008:358-359.

4. Janot $\mathrm{Fr}$.

Les gestes

de l'embaumeur dans la cavité buccale. Actes. Société française d'histoire de l'art dentaire 2009;14:63-66.

5. Janot Fr, Fass D. Le corps d'une "prophétesse» ? Mitteilungen des Deutschen Archäologischen Institut Kairo. Berlin (à paraître).

6. Bombardier $\mathrm{P}$. Les problèmes bucco-dentaires des instrumentistes à vents.

Impératifs à respecter

lors des traitements.

Thèse chir. dent. Nancy, 1982.

7. Molleson T.

Des ossements

révélateurs.

Pour la Science

1994;204:57-60.

8. Janot $\mathrm{Fr}$.

Une occupante inattendue

de la pyramide

du roi Pépy ler.

Bulletin de I'Institut français

d'archéologie orientale 2000;100:347-369.

9. Janot $\mathrm{Fr}$.

Odontologie et archéologie égyptienne :

une femme cordonnière retrouvée sur la pyramide du roi Pépy ler à Saqqara.

Bulletin de l'Académie nationale de chirurgie dentaire 2003:46:35-42.

10. Piette E., Golberg M. La dent normale et pathologique. Paris : De Boeck, 2001.

11. Benmehdi S.

Érosions dentaires :

une pathologie

en progression.

Clinic 2008;29:577-585.

12. Leluhard $\mathrm{R}$.

Art bakongo,

insignes de pouvoir,

le sceptre.

Arnouville : 1998

13. Viala A, Botta $A$.

Toxicologie.

Paris : Tec \& Doc/Lavoisier 2005:833.

14. Gayet A.

Notice des objets recueillis

à Antinoë

(fouilles de 1906-1907).

Paris 1907:45-47.
15. Legrain G.

Le logement et transport

des barques sacrées

et des statues des dieux

dans quelques temples

égyptiens.

Bulletin de I'Institut français

d'archéologie orientale

1917:13:1-76.

16. Cerny J.

Questions adressées aux oracles.

Bulletin de I'Institut français d'archéologie orientale 1935;35:41-58.

17. Anasgnostou-Callas B.

"Justice" oraculaire

l'Égypte hellénistique et romaine.

Rev hist droit

1998;76/1:1-16.

18 Papaconstantinou A

Oracles chrétiens

dans l'Égypte

byzantine :

le témoignage

des papyrus.

Zeitschrift für Papyrologie

und Epigraphik

1994;104:281-286.

19 Valbelle D, Husson G.

Les questions

oraculaires

d'Égypte :

histoire de la recherche,

nouveautés

et perspectives.

In Clarisse W, Schoors A,

Willens $\mathrm{H}$. ed.

Egyptian Religion.

The last Thousand Years,

Studies dedicated

to the Memory

of Jan Quaegebeur,

Orientalia lovaniensa

analecta.

Leuven: 1998;85/2:1055-1071. 
20. Papini L.

Biglietti oracolari in copto

dalla necropoli

Nord di Antinoe.

In : Orlandi T, Wisse F ed., Acts of the Second International Congress of Coptic Study 22-26 September 1980. Roma 1985:245-255.
21. Trauneker $\mathrm{Cl}$. L'appel au divin : la crainte des dieux et les serments du temple. In : Heintz J.-G. éd., Oracles et prophéties dans l'Antiquité. Actes du Colloque de Strasbourg
15 au 17 juin 1995 ,

Paris 1997:35-54.

22. Hebrard J.

L'apport de la science dans la preuve pénale. Bulletin de l'Académie de Lorraine des Sciences 2005;44:41-45.

\section{SUMMARY}

\section{The Forensic Proof on Dental Organ of the Mummy of Grenoble}

Francis JANOT,

Jean-Paul ARTIS,

Henry COUDANE,

Pierre BRAVETTI

\section{Keywords \\ forensic odontology \\ - egyptology \\ - forensic proofs \\ - mummy of Grenoble}

By its specificities, forensic odontology is an inescapable discipline in the study of bodies of the disappeared civilizations. In Ancient Egypt, the indications given by the dental organ can become real forensic proofs and indicate a profession. Such is the case for the mummy of Grenoble. 